\title{
PROPOSTA DE UMA VERSÃO BRASILEIRA PARA A ESCALA ADCS-CGIC
}

\author{
Paulo H.F. Bertolucci' ${ }^{1}$ Ricardo Nitrini ${ }^{2}$
}

\begin{abstract}
RESUMO - Com o crescente volume de pesquisas sobre novas terapias para as demências, em particular a doença de Alzheimer, tornou-se evidente que a avaliação mais tradicional, centrada nas alterações da cognição, não seria suficiente para detectar algumas das alterações significativas que novos tratamentos podem trazer. Deste modo, foram acrescentadas escalas para avaliar alterações do comportamento e da dependência funcional. Ainda assim, restavam mudanças, percebidas pelos familiares, que poderiam não ser captadas por essas escalas. As escalas de impressão clínica de mudança (CIGIC) baseiam-se nas informações fornecidas pelo informante, no que é observado durante a consulta, e, se necessário, em breves testes de cognição. Apresentamos a versão de uma escala deste tipo, largamente utilizada, desenvolvida para a doença de Alzheimer (ADCS-CIGIC), que foi obtida pelo método recomendado de tradução, retro-tradução e versão final por consenso.
\end{abstract}

PALAVRAS-CHAVE: escalas de avaliação, CGIC, doença de Alzheimer, demência.

\begin{abstract}
A Brazilian Portuguese version for the ADCS-CGIC scale
ABSTRACT - Given increasing research into new therapies for dementia, especially for Alzheimer disease, it has become clear that traditional methods of evaluation, centered on cognition, have proved insufficient. Thus, scales for behavioral disturbances and activities of daily living have been added. Nevertheless, some observations of clinical significant changes, as reported by caregivers, could be overlooked. Clinician's impression of global change scales (CIGIC) are based on broader information and may detect more subtle changes. Typically they take into consideration caregiver information, that which is seen by the health professional during the interview, and may also include brief cognitive tests. We present a Brazilian Portuguese version of a widely used scale of this type, specifically designed for Alzheimer disease (ADCS-CGIC), being the result of the recommended method of translation, back-translation and version by panel consensus.
\end{abstract}

KEY WORDS: evaluation scales, CGIC, Alzheimer disease, dementia.

As demências e, em particular, a doença de Alzheimer (DA), costumam ser definidas em termos de alteração cognitiva. De fato, os dois critérios diagnósticos mais usados para a DA dão ênfase a estes aspectos da doença. Assim, o critério dos Institutos Nacionais de Saúde dos Estados Unidos - Associação de Doença de Alzheimer e Doenças Relacionadas (NINCDS-ADRDA) ${ }^{1}$ considera como provável DA o declínio na memória e pelo menos numa outra área da cognição, desde que afastadas outras causas de demência e outros distúrbios com impacto sobre o sistema nervoso central. O Manual de Diagnóstico e Estatística da Associação Americana de Psiquiatria, em sua última versão, DSM-IV², do mesmo modo, requer prejuízo da memória e pelo me- nos de uma outra área da cognição, mas adicionalmente é necessário que estes déficits interfiram no funcionamento social e ocupacional. Este último aspecto é importante, porque incorpora o conceito de declínio a partir de um nível de funcionamento prévio, algo que pode ser perdido em uma avaliação exclusivamente cognitiva. Os testes cognitivos são extremamente úteis para o diagnóstico das demências, e já existe extensa literatura sobre a sensibilidade de diferentes testes, tanto para o diagnóstico precoce da DA, como para o valor preditivo da probabilidade de demenciação a curto prazo. Mostraram seu valor neste sentido tanto testes de rastreio, como o Mini-exame do Estado Mental3 ${ }^{3}$, quanto sub-testes de baterias completas, como o item

\footnotetext{
1Professor Adjunto, Departamento de Neurologia e Neurocirurgia, Escola Paulista de Medicina, Universidade Federal de São Paulo,(EPMUNIFESP) São Paulo SP, Brasil, 2Professor Associado, Departamento de Neurologia, Faculdade de Medicina, Universidade de São Paulo, (FMUSP) São Paulo, SP, Brasil.

Recebido 20 Fevereiro 2003, recebido na forma final 28 Março 2003. Aceito 14 Abril 2003.

Dr. Paulo H.F. Bertolucci - Disciplina de Neurologia, UNIFESP - Rua Botucatu 740 - 04023-900 São Paulo SP - Brasil. E-mail-paulohb@neuro.epm.br
} 
de similaridades da Escala Wechsler de Inteligência para Adultos (WAIS). Apesar de sua utilidade, estes procedimentos podem apresentar falhas em dois sentidos: uma pessoa pode apresentar mau desempenho nos testes sem que isso necessariamente signifique declínio e possibilidade de demência, como ocorre, por exemplo, ao avaliar pessoas com baixa escolaridade ${ }^{4,5}$ ou embotamento intelectual. Por outro lado, uma pessoa altamente intelectualizada pode estar claramente apresentando declínio em relação a seu funcionamento prévio, e ainda assim apresentar desempenho dentro do esperado ${ }^{6}$. Isto não significa que os testes de avaliação não sejam úteis no diagnóstico das demências, mas sim que pode haver falhas em determinadas situações e que podem ser necessários outros métodos.

Além dos aspectos diagnósticos, as escalas servem também para avaliar o efeito de intervenções terapêuticas. Aqui, o uso de escalas que focalizam as alterações na atividade funcional e no comportamento são fundamentais, pois estas são as áreas que tornam as demências mais desgastantes para o cuidador. Por esta razão, a agência americana responsável pela aprovação de novas drogas (Food and Drug Administration - FDA) requer evidência de efeito em uma escala objetiva (tipicamente a bateria de avaliação cognitiva da Alzheimer Disease Assessment Scale - ADAS-cog) e uma escala que leve em consideração atividade funcional e comportamento ${ }^{7}$. As escalas de impressão de mudança, desde os primeiros estudos de drogas para a DA, mostraram-se os indicadores mais sensíveis de mudanças, quando comparadas com outros instrumentos ${ }^{8}$.

Um aspecto fundamental no planejamento destas escalas é que elas devem medir mudanças clinicamente significativas, diferentemente, por exemplo, das escalas cognitivas, que, em princípio, detectam qualquer mudança. Assim, o clínico que indica mudança neste tipo de escala em um indivíduo, está indicando "mudança clinicamente significativa e definida"9. Ainda em relação à estrutura da escala, quando mais detalhada ela se tornar, maior a chance de detectar mudanças mais sutis, que podem não ter significado clínico. Portanto o sucesso destas escalas depende de um equilíbrio entre: serem genéricas, mas não a ponto de permitir grau excessivo de subjetividade por parte do investigador e variação muito ampla entre examinadores; e detalhadas, mas não tanto que se tornem "métricas", como as escalas objetivas usadas habitualmente na avaliação cognitiva, que podem detectar mesmo modificações muito pequenas. Deste modo, o maior desafio em relação às escalas de impressão clínica de mudança tem sido manter sua capacidade de descartar modificações sem relevância, mas sem perder a reprodutibilidade.

O uso de escalas de impressão de mudança nas demências começou os primeiros estudos de avaliação do efeito da tacrina ${ }^{10}$, onde a melhora detectada por um instrumento deste tipo foi selecionada como um dos objetivos alvo. Com a posterior multiplicação de ensaios clínicos, várias escalas diferentes passaram a ser usadas, o que levou o FDA a especificar ainda mais os requisitos para escalas de impressão global de mudança. Neste sentido surgiu a Clinical Interview Based Impression of Change (CIBIC-plus), que avalia o estado clinico global do paciente, em comparação com a linha de base, a partir de entrevista com a própria pessoa e com um cuidador. Mas, ainda assim, a variabilidade dos resultados levantava sérias dúvidas sobre os resultados deste tipo de escala.

A necessidade de realizar estudos em larga escala de novas drogas para demência levou a que, em cooperação com o FDA, fosse criada uma nova escala para avaliar o efeito da tacrina, a Clinician Interview Based Impression (Impressão Baseada na Entrevista Clínica - CIBI) ${ }^{11}$. Diferente de escalas anteriores, esta define oito itens específicos que devem ser abordados e julgados de acordo com uma escala variando de 1 (muito pior) a 7 (muito melhor). Quase ao mesmo tempo, o Instituto Nacional para o Envelhecimento financiava o Alzheimer's Disease Cooperative Study, consórcio multi-cêntrico entre cujas responsabilidades estava a de "avaliar e, se necessário, melhorar os instrumentos existentes para testar drogas na doença de Alzheimer" 9 . Foi como parte deste esforço que a escala ADCS-CGIC (Alzheimer's Disease Cooperative Study - Clinical Global Impression of Change) foi criada. A escala avalia uma série de domínios, para os quais são salientados pontos específicos que podem ser úteis como indicadores de mudança clínica salientados. Considerando a utilidade desta escala, decidimos fazer sua versão para o português.

Método para a tradução: A versão original do ADCS-CGIC ${ }^{12}$ foi traduzida para o português por uma empresa especializada em tradução de textos médicos. Esta versão foi em seguida traduzida de volta para o inglês, de modo independente, por dois neurologistas experientes em escalas para demência e fluentes em inglês. As diferenças de tradução foram 
identificadas e discutidas em painel, considerando as razões para as diferenças e cotejando-as com a forma original em inglês, chegando-se à forma apresentada em anexo.

Método de aplicação da escala: É determinado o estado basal, a partir de entrevistas com o paciente e um informante, sendo considerado informante qualquer pessoa que conheça bem e conviva com o paciente, geralmente o cônjuge ou um filho. É preenchida a primeira parte da escala (CIBIS basal - anexo 1) , cujas informações vão servir de referência para determinar, em avaliações posteriores, se houve mudança ou não. Esta avaliação baseia-se na observação de 15 domínios da cognição, comportamento, funcionamento social e atividades da vida diária. Apesar de haver poucos requisitos formais em relação a esta primeira entrevista, é recomendável a avaliação do estado mental, que pode ser feita com testes breves.

Uma vez que a entrevista inicial seja concluída, deve ser registrada a fonte das informações obtidas, que poderá ser a entrevista com o paciente, entrevista com o informante, testes neuropsicológicos, ou outras.

Em cada avaliação subseqüente, o examinador deverá usar um método semelhante para entrevistar o paciente e o informante, o que deve ser realizado separadamente. Além das normas da entrevista de base, o examinador deve ser instruído para não se basear em outros instrumentos de estudo e para não perguntar sobre a impressão do paciente e do informante sobre os efeitos do tratamento ou sobre efeitos colaterais. Também não deve ser discutido o nível de funcionamento do paciente com outros profissionais de saúde que estejam atendendo o paciente. Em estudos experimentais, o avaliador faz duas avaliações de mudança, uma para cada entrevista e são obtidos dois escores, de modo que seja possível determinar qual foi a contribuição do paciente e do informante para o escore final do ADCS-CGIC (anexo 2) em cada visita.

O escore do ADCS-CGIC é feito a partir de uma escala com a seguinte graduação:

1 - melhora marcada; 2 - melhora moderada; 3 melhora mínima; 4 - sem mudança; 5 - piora mínima; 6 - piora moderada; 7 - piora marcada.

Enquanto o tempo para o preenchimento do CIBIS basal pode ser variável, não havendo restrição alguma neste sentido, a obtenção de informações e preenchimento do ADCS-CGIC estão limitados a 20 minutos $^{15}$.

Considerações finais: Embora a primeira impressão possa ser de que a escala permite um grau excessivo de subjetividade, estudos anteriores ${ }^{9}$, acompanhando prospectivamente pacientes com DA, mostraram: confiabilidade tanto a curto como a longo prazo; correlação modesta com outras escalas, como o Mini-exame do Estado Mental ${ }^{13}$ e a escala $\mathrm{GDS}^{14}$. Deve ser lembrado que estas são escalas para avaliar gravidade da doença, e não mudança. Seu uso continuado em protocolos de avaliação de terapêutica mostra que este é um instrumento confiável e de fácil aplicação.

\section{REFERÊNCIAS}

1. McKhann G, Drachman D, Folstein M, Katzman R, Price D, Stadlan EM. Clinical diagnosis of Alzheimer's disease: report of the NINCDSADRDA Work Group under the auspices of Department of Health and Human Services Task Force on Alzheimer's Disease. Neurology 1984;34:939-944.

2. American Psychiatric Association. Diagnostic and Statistical Manual of Mental Disorders, 4 Ed. Washington, DC: American Psychiatric Association, 1994.

3. Commenges D, Gagnon M, Letenneur L, Dartigues J-F, Barbeger-Gateau $\mathrm{P}$, Salamon R. Improving screening for dementia in the elderly using Mini-Mental State Examination subscores, Benton's Visual Retention Test and Isaacs Set Test. Epidemiology 1992;3:185-188.

4. Bertolucci PHF, Brucki SMD, Campacci SR, Juliano Y. O Mini-exame do Estado Mental em uma população geral: impacto da escolaridade. Arq Neuropsiquiatr 1994;52:1-7.

5. Fabrigoule C, Lafont S, Letteneur L, Dartigues J-F. WAIS similarities subtest performances as predictors of dementia in elderly community residents. Brain Cog 1996;30:323-326.

6. Tombaugh TN, McIntyre NJ. The Mini-Mental State Examination: a comprehensive review. JAGS 1992;40:922-935.

7. Leber P. Guidelines for the clinical evaluation of antidementia drugs. Washington DC: Food and Drug Administration, 1990.

8. Lehman E. Practicable and valid approach to evaluate the efficacy of nootropic drugs by means of rating scales. Pharmacopsychiatry 1984;17:71-75.

9. Schneider L, Olin JT, Doody RS et al. Validity and reliability of the Alzheimer's disease Cooperative Study: clinical global impression of change (ADCS-CGIC). In Becker R, Giacobini E (Eds). Alzheimer disease: from molecular biology to therapy. Boston. Birkhäuser, 1996:1-18.

10. Summers WK, Majovski LV, Marsh GM, Tachiki K, Kling A. Oral tetrahydroaminoacridine in long-term treatment of senile dementia Alzheimer type. N Engl J Med 1986;315:1241-1245.

11. Knopman DS, Knapp MI, Gracon SI, Davis CS. The Clinician Interview Based Impression (CIBI): a clinician's global change rating scale in Alzheimer's disease. Neurology 1994;44:2315-2321.

12. Ferris SH, Mackell JA, Mohs R, et al. A multicenter evaluation of new treatment efficacy instruments for Alzheimer's disease clinical trials: overview and general results. The Alzheimer Disease Cooperative Study. Alzh Dis Assoc Disord 1997;11:S1-12.

13. Folstein MF, Folstein S, McHugh PR. Mini-mental state: a practical guide for grading the cognitive state of patients for the clinician. J Psychiatr Res 1975;12:189-198.

14. Reisberg B, Ferris SH, deLeon MJ, Crook T. The global deterioration scale for the assessment of primary degenerative dementia. Am J Psychiatr 1982;139:1136-1139.

15. Schneider LS, Olin JT, Doody RS, et al. Validity and reliability of the Alzheimer's Disease Cooperative Study-Clinical Global Impression of Change. Alzh Dis Assoc Disord 1991;11(Sup. 2):S22-S32. 


\section{ANEXO 1}

ESTUDO / PROTOCOLO N ${ }^{\circ}$

$\mathrm{N}^{\circ}$ de randomização:

\section{IMPRESSÃO DO MÉDICO SOBRE A GRAVIDADE DA DOENÇA COM BASE EM ENTREVISTA (CIBIS) CIBIS BASAL \\ Instruções para avaliação}

Visita $\mathrm{n}^{\circ}$

A entrevista semi-estruturada baseia-se, em parte, no instrumento ADCS de Impressão Global de Alteração que foi derivado sob concessão do National Institute on Aging nos EUA. Ele identifica quatro categorias principais para avaliação: GERAL, ESTADO MENTAL/COGNITIVO, COMPORTAMENTO E ATIVIDADES DA VIDA DIÁRIA.

Cada uma destas quatro categorias está subdividida em DOMínIOS.

\begin{tabular}{llll}
\hline GERAL & ESTADO MENTAL/COGNITIVO & COMPORTAMENTO & ATIVIDADES DA VIDA DIÁRIA \\
\hline História relevante & $\begin{array}{l}\text { Prontidão/Alerta } \\
\text { Atenção/Concentração }\end{array}$ & Conteúdo do pensamento & $\begin{array}{l}\text { Capacidades funcionais } \\
\text { básicas e complexas } \\
\text { (atividades instrumentais) } \\
\text { Observação/ Avaliação }\end{array}$ \\
& Orientação & Alucinações/ & Delírios/llusões \\
& Comportamento/Humor & Sono/Apetite \\
& Lemória & Neurológico/ & \\
& Praxias & Atividade Psicomotora & \\
& Julgamento/Resolução de & \\
problemas/ Auto-percepção & \\
& (Insight) & \\
\hline
\end{tabular}

Cada DOMíNIO é avaliado através de alguns instrumentos de sondagem. Para todos os DOMíNIOS, são sugeridos alguns instrumentos de sondagem. 0 entrevistador é estimulado a escolher instrumentos adicionais, para aumentar a compreensão da entrevista.

\section{IMPRESSÃO DO MÉDICO SOBRE A GRAVIDADE DA DOENÇA COM BASE EM ENTREVISTA (CIBIS) Página 1 de 6 ENTREVISTA COM O PACIENTE E O CUIDADOR

\author{
Instruções resumidas
}

Esta entrevista basal é para ser realizada primeiro com o paciente e depois com o cuidador. Não é necessário que os DOMíNIOS sejam avaliados em uma ordem determinada. Todas as observações devem ser anotadas com detalhes suficientes para facilitar a avaliação da alteração nas avaliações subsequentes. Além disso, devem ser realizadas algumas avaliações de Estado Mental/Cognitivo. A gravidade deve ser julgada com as informações obtidas de todas as fontes relevantes, i.e., paciente, cuidador, membros da equipe do estudo etc. Adicionalmente, os resultados de todas as avaliações psicométricas basais e de seleção devem ser anotadas na última página deste módulo e consideradas na avaliação do escore CIBIS.

A impressão da gravidade da doença é anotada na ficha clínica do estudo, na página específica de CIBIS, usando a escala fornecida.

\begin{tabular}{lll}
\hline DOMíNIO & INSTRUMENTOS DE SONDAGEM & OBSERVAÇÕES \\
\hline História relevante & $\begin{array}{l}\text { Eventos clínicos relevantes recentes, } \\
\text { doenças do paciente, eventos sociais ou } \\
\text { pessoais significativos }\end{array}$ & Paciente \\
$\begin{array}{l}\text { Cuidador } \\
\text { Observação/Avaliação }\end{array}$ & $\begin{array}{l}\text { Aparência - limpeza e higiene geral, } \\
\text { roupas (adequação ao clima, limpeza, } \\
\text { combinação de cores/estampas, } \\
\text { maneira como abotoou) }\end{array}$ & Paciente \\
\hline
\end{tabular}


IMPRESSÃO DO MÉDICO SOBRE A GRAVIDADE DA DOENÇA COM BASE EM ENTREVISTA (CIBIS) Página 2 de 6 ESTADO MENTAL/COGNITIVO (Exame estruturado, se utilizado:

...)

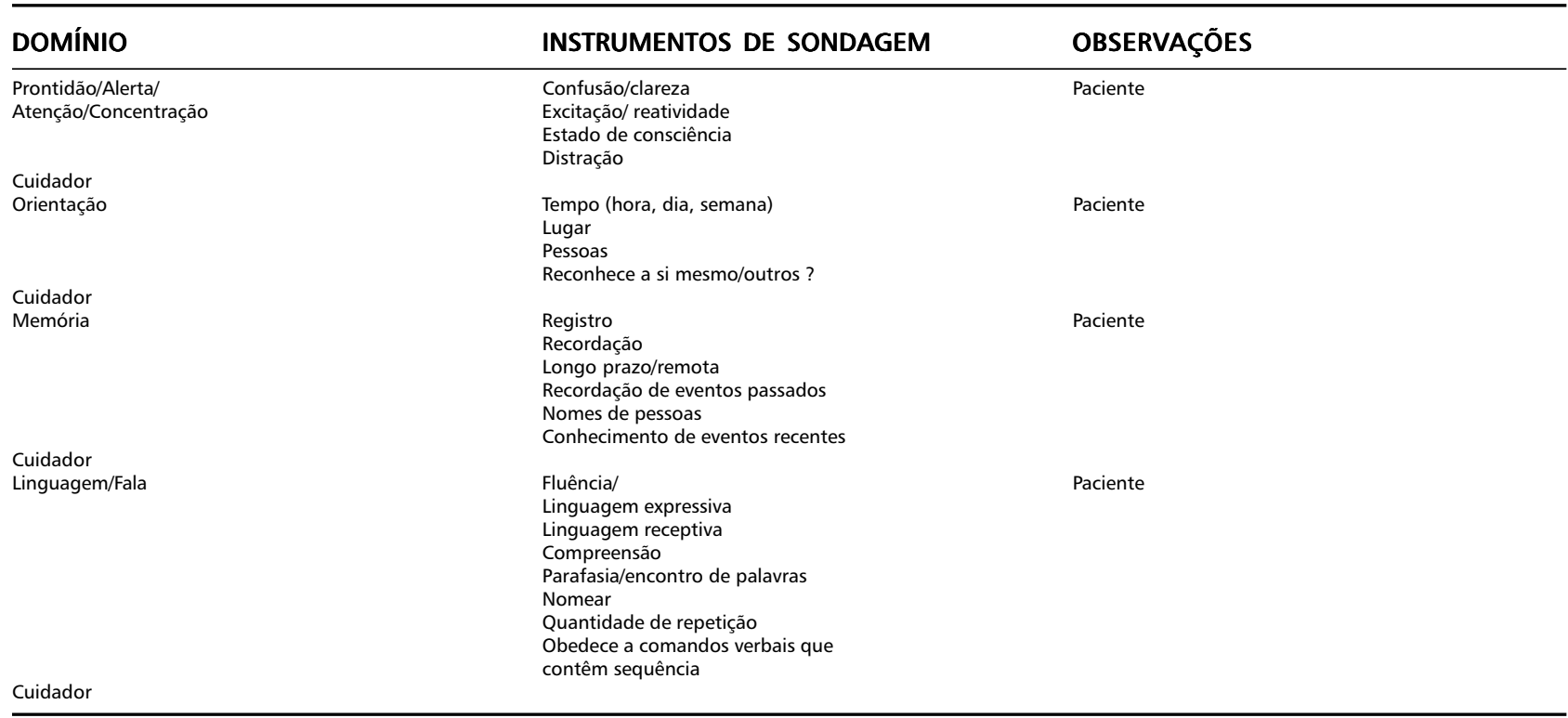

IMPRESSÃO DO MÉDICO SOBRE A GRAVIDADE DA DOENÇA COM BASE EM ENTREVISTA (CIBIS) Página 3 de 6 ESTADO MENTAL/COGNITIVO (continuação)

\begin{tabular}{|c|c|c|}
\hline DOMÍNIO & INSTRUMENTOS DE SONDAGEM & OBSERVAÇÕES \\
\hline Praxias & $\begin{array}{l}\text { Praxia construtiva } \\
\text { (i.e., desenhar um cubo tridimensional, } \\
\text { mostrador de relógio com horas) } \\
\text { Praxia ideatória } \\
\text { Praxia ideomotora/imitação }\end{array}$ & Paciente \\
\hline $\begin{array}{l}\text { Cuidador } \\
\text { Julgamento/ } \\
\text { Solução de problemas/ } \\
\text { Auto-percepção (Insight) }\end{array}$ & $\begin{array}{l}\text { Comportamento do paciente em } \\
\text { situações que necessitam julgamento } \\
\text { (p.e., falta de eletricidade em casa, } \\
\text { ficar trancado fora de casa etc) } \\
\text { Consciência de si mesmo (i.e., de erros, } \\
\text { comportamento impróprio, julgamento } \\
\text { deficiente) } \\
\text { Tentativas de corrigir erros } \\
\text { (i.e., de raciocínio, erros) }\end{array}$ & Paciente \\
\hline Cuidador & & \\
\hline
\end{tabular}

IMPRESSÃO DO MÉDICO SOBRE A GRAVIDADE DA DOENÇA COM BASE EM ENTREVISTA (CIBIS) Página 4 de 6 COMPORTAMENTO

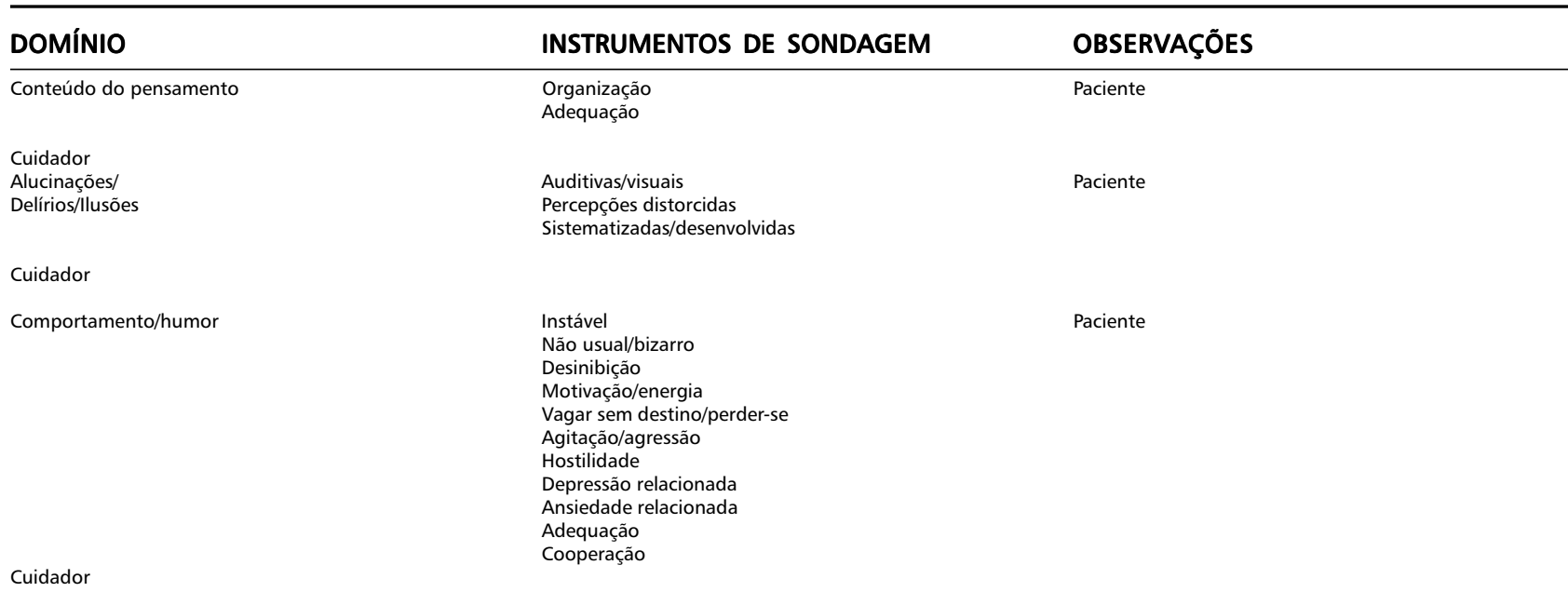




$\begin{array}{lll}\text { Sono/Apetite } & \begin{array}{l}\text { Distúrbios do sono } \\ \text { Insônia (tipo?) } \\ \text { Atividade noturna } \\ \text { Sonolência/pouco sono } \\ \text { Apetite/alteração de peso } \\ \text { Preferências alimentares }\end{array} \\ \text { Cuidador } & \begin{array}{l}\text { Paciente } \\ \text { Neurológico } \\ \text { Atividade psicomotora }\end{array} & \begin{array}{l}\text { Postura/marcha } \\ \text { Distúrbios do movimento } \\ \text { Comportamento motor não usual/ } \\ \text { Movimentos de marcar passos (pacing) } \\ \text { Padrões diários de atividade }\end{array} \\ & \end{array}$

IMPRESSÃO DO MÉDICO SOBRE A GRAVIDADE DA DOENÇA COM BASE EM ENTREVISTA (CIBIS) Página 5 de 6 ATIVIDADE DA VIDA DIÁRIA

\begin{tabular}{|c|c|c|}
\hline DOMÍNIO & INSTRUMENTOS DE SONDAGEM & OBSERVAÇÕES \\
\hline $\begin{array}{l}\text { Capacidade funcional básica e complexa } \\
\text { (atividades instrumentais) }\end{array}$ & $\begin{array}{l}\text { Mobilidade (caminhar, dirigir, usar o } \\
\text { transporte público, habilidade em } \\
\text { encontrar o caminho) } \\
\text { Higiene/cuidado pessoal } \\
\text { Vestir-se/escolher roupas } \\
\text { Alimentar-se sozinho, preparar a comida } \\
\text { Realizar incumbências } \\
\text { Compras } \\
\text { Tarefas domésticas } \\
\text { Hobbies e atividades pessoais } \\
\text { (i.e., ler, fazer jardinagem, pintar, } \\
\text { ver televisão) } \\
\text { Finanças } \\
\text { Telefone }\end{array}$ & Paciente \\
\hline \multicolumn{3}{|l|}{ Cuidador } \\
\hline Função social & $\begin{array}{l}\text { Participa de: } \\
\text { Jogos com outras pessoas (i.e., cartas, } \\
\text { xadrez, dama) } \\
\text { Interações sociais } \\
\text { Atividades domésticas (i.e., refeições } \\
\text { familiares) } \\
\text { Atividades externas (i.e., visita um amigo } \\
\text { em casa, janta fora, pratica esportes } \\
\text { com os amigos) } \\
\text { Atividades comunitárias (i.e., religiosas, } \\
\text { profissionais) } \\
\text { Frequenta eventos (i.e., teatro, esportes, } \\
\text { cinema) } \\
\text { Independência } \\
\text { Fica desamparado }\end{array}$ & Paciente \\
\hline Cuidador & & \\
\hline
\end{tabular}

Notas, comentários, resumo das

Informação de outras fontes: afirmações:

Nome do examinador:

Iniciais do examinador:

Hora em que o teste foi aplicado:

IMPRESSÃO DO MÉDICO SOBRE A GRAVIDADE DA DOENÇA COM BASE EM ENTREVISTA (CIBIS) Página 6 de 6 


\begin{abstract}
ANEXO 2
ESTUDO / PROTOCOLO No Iniciais do paciente:

No de randomização:

Data:

Visita $\mathrm{n}^{\circ}$

ESTUDO COOPERATIVO DA DOENÇA DE ALZHEIMER - IMPRESSÃO CLÍNICA GLOBAL DE ALTERAÇÃO (ADCS-CGIC)

ANTES DE REALIZAR QUALQUER ADCS-CGIC, O MÉDICO DEVE ESTUDAR O CIBIS BASAL PARA FAMILIARIZAR-SE COM O PERFIL DA DOENÇA DO PACIENTE.

O ADCS-CGIC é realizado através de entrevistas separadas, primeiro com o paciente e depois com o cuidador.

Após as entrevistas, o médico deve decidir sozinho se as condições do paciente alteraram-se ou não de acordo com a linha basal. 0 médico não deve consultar outros membros da equipe de avaliação do paciente nem olhar o resultado de outros procedimentos ou testes realizados durante qualquer Visita Clínica que não seja a da linha basal. O médico deve evitar perguntar a opinião do paciente ou do cuidador que pode influenciar a escala do ADCS-CGIC, como opiniões relacionadas às alterações nos sintomas periféricos ou a evidência de efeitos colaterais (experiências adversas ao medicamento). No início de cada entrevista, o médico deve alertar a ambos que responderão ao questionário para que evitem fornecer tais informações.

A impressão da alteração é anotada na ficha clínica do estudo, na página específica de ADCS-CGIC usando a escala de 7 itens que ali está. Primeiro entrevista-se o paciente, e depois o cuidador antes de fazer a avaliação. Depois que o escore estiver estabelecido, a página específica ADCS-CGIC da ficha clínica para esta visita clínica deve ser entregue ao Coordenador do Estudo para inclusão na ficha clínica do paciente. Assim que esta página específica tiver sido liberada pelo(a) avaliador(a) do ADCS-CGIC, ele(a) não deve mais consulta-la novamente.

O tempo estimado para completar a entrevista ADCS-CGIC é aproximadamente 20 minutos para cada entrevista - com o paciente e com o cuidador.
\end{abstract}

ESTUDO COOPERATIVO DA DOENÇA DE ALZHEIMER- IMPRESSÃO CLíNICA GLOBAL DE ALTERAÇÃO (ADCS-CGIC) Página 1 de 9

Instruções:

Reveja a folha de instrução e o CIBIS Basal antes de realizar esta entrevista. Anote as informações recebidas do paciente nos espaços fornecidos abaixo. É importante evitar qualquer discussão sobre os efeitos colaterais ou a opinião do cuidador se o paciente está usando o medicamento ativo. Não é sugerido nenhum formato nem ordem particulares para a entrevista.

GERAL

\begin{tabular}{lll}
\hline DOMíNIO & INSTRUMENTOS DE SONDAGEM & OBSERVAÇÕES \\
\hline História no intervalo & Eventos clínicos desde a visita basal \\
Observação/Avaliação & Aparência \\
\hline
\end{tabular}

ESTUDO COOPERATIVO DA DOENÇA DE ALZHEIMER- IMPRESSÃO CLÍNICA GLOBAL DE ALTERAÇÃO (ADCS-CGIC) Página 2 de 9 ESTADO MENTAL/COGNITIVO (Exame estruturado, se utilizado: .................. )

\begin{tabular}{lll}
\hline DOMíNIO & INSTRUMENTOS DE SONDAGEM & OBSERVAÇÕES \\
\hline Prontidão/Alerta/ & Confusão/clareza \\
Atenção/Concentração & Excitação \\
& Reação/estado de consciência \\
Orientação & Tempo \\
& Lugar \\
& Pessoa \\
Memória & Registro \\
& Recordação \\
& Longo prazo/remoto
\end{tabular}




$\begin{array}{ll}\text { Linguagem/Fala } & \text { Fluência/ } \\ & \text { Linguagem expressiva } \\ & \text { Linguagem receptiva } \\ & \text { Compreensão } \\ & \text { Parafasia/encontrar palavras } \\ \text { Praxias } & \text { Praxia construtiva } \\ & \text { Praxia ideatória } \\ & \text { Praxia ideomotora/imitação }\end{array}$

Julgamento/Solução de problemas

ESTUdO COOPERATIVO DA DOENÇA DE ALZHEIMER- IMPRESSÃo CLínICA GLOBAL DE ALTERAÇÃo (ADCS-CGIC) Página 3 de 9 COMPORTAMENTO

\begin{tabular}{|c|c|c|}
\hline DOMÍNIO & INSTRUMENTOS DE SONDAGEM & OBSERVAÇÕES \\
\hline \multirow[t]{2}{*}{ Conteúdo do pensamento } & Organização & \\
\hline & Adequação & \\
\hline Alucinações/ & Auditivas/visuais & \\
\hline \multirow[t]{2}{*}{ Delírios/llusões } & Percepções distorcidas & \\
\hline & Sistematizadas/desenvolvidas & \\
\hline \multirow[t]{8}{*}{ Comportamento/humor } & Instável & \\
\hline & Motivação/energia & \\
\hline & Agitação/agressão & \\
\hline & Hostilidade & \\
\hline & Depressão/Ansiedade relacionada & \\
\hline & Desinibição & \\
\hline & Adequação & \\
\hline & Cooperação & \\
\hline \multirow[t]{3}{*}{ Sono/Apetite } & Distúrbios do sono & \\
\hline & Atividade noturna & \\
\hline & Apetite/alteração de peso & \\
\hline \multirow[t]{4}{*}{ Atividade psicomotora/ neurológica } & Atividade motora global & \\
\hline & Postura/marcha & \\
\hline & Distúrbios do movimento & \\
\hline & Comportamento motor não usual & \\
\hline
\end{tabular}

ESTUdO COOPERATIVO DA DOENÇA DE ALZHEIMER- IMPRESSÃo CLínICA GLOBAL DE ALTERAÇÃo (ADCS-CGIC) Página 4 de 9 FUNÇÕES

\begin{tabular}{|c|c|c|}
\hline DOMÍNIO & INSTRUMENTOS DE SONDAGEM & OBSERVAÇÕES \\
\hline Capacidade funcional & Mobilidade & \\
\hline básica e complexa & Higiene/cuidados & \\
\hline \multirow[t]{3}{*}{ (atividades instrumentais) } & Vestir-se & \\
\hline & Alimentar-se sozinho & \\
\hline & Fazer compras & \\
\hline \multirow[t]{2}{*}{ Função social } & Interações & \\
\hline & Atividades comunitárias & \\
\hline Notas e comentários: & & \\
\hline
\end{tabular}


ESTUdO COOPERATIVO DA DOENÇA DE ALZHEIMER- IMPRESSÃo CLÍNICA GLOBAL DE ALTERAÇÃO (ADCS-CGIC) Página 5 de 9 ENTREVISTA COM O CUIDADOR

\section{Instruções}

Reveja a folha de instrução e o CIBIS basal antes de realizar esta entrevista. Anote as informações recebidas do cuidador nos espaços fornecidos abaixo. É importante evitar qualquer discussão sobre os efeitos colaterais ou sobre a opinião do cuidador se o paciente está usando o medicamento ativo. Não é sugerido nenhum formato nem ordem particulares para a entrevista.

\section{GERAL}

\begin{tabular}{lll}
\hline DOMíNIO & INSTRUMENTOS DE SONDAGEM & OBSERVAÇÕES \\
\hline História no intervalo & Eventos clínicos desde a visita basal \\
& Alterações? \\
& Doenças? \\
& Efeitos? \\
Aderência & Aderência ao protocolo \\
& Problemas \\
\hline
\end{tabular}

ESTUDO COOPERATIVO DA DOENÇA DE ALZHEIMER- IMPRESSÃO CLÍNICA GLOBAL DE ALTERAÇÃO (ADCS-CGIC) Página 6 de 9 ESTADO MENTAL/COGNITIVO

\begin{tabular}{|c|c|c|}
\hline DOMíNIO & INSTRUMENTOS DE SONDAGEM & OBSERVAÇÕES \\
\hline Prontidão/Alerta/ & Confusão/clareza & \\
\hline \multirow[t]{2}{*}{ Atenção/Concentração } & Excitação /reação & \\
\hline & Estado de consciência & \\
\hline \multirow[t]{5}{*}{ Orientação } & Relações temporais & \\
\hline & Viagem & \\
\hline & Encontra seu caminho? & \\
\hline & Reconhece a si mesmo/aos outros/objetos & \\
\hline & Reage de forma apropriada? & \\
\hline \multirow[t]{3}{*}{ Memória } & Recordação & \\
\hline & Memória & \\
\hline & Eventos passados & \\
\hline \multirow[t]{7}{*}{ Linguagem } & Expressão & \\
\hline & Compreensão & \\
\hline & Encontrar palavras & \\
\hline & Nomear & \\
\hline & Quantidade de repetição & \\
\hline & Obedece a comandos que contêm & \\
\hline & seqüência & \\
\hline \multirow[t]{5}{*}{ Atividade motora } & Atividade global & \\
\hline & Padrões diários & \\
\hline & Perambulação & \\
\hline & Distúrbios do movimento & \\
\hline & Movimentos não usuais & \\
\hline \multirow[t]{2}{*}{ Julgamento/Solução de problemas } & Comportamento do paciente em situações & \\
\hline & que requerem julgamento & \\
\hline
\end{tabular}


ESTUdO COOPERATIVO DA DOENÇA DE ALZHEIMER- IMPRESSÃO CLíNICA GLOBAL DE ALTERAÇÃO (ADCS-CGIC) Página 7 de 9 COMPORTAMENTO

\begin{tabular}{lll}
\hline DOMÍNIO & INSTRUMENTOS DE SONDAGEM & OBSERVAÇÕES \\
\hline Conteúdo do pensamento & Organização \\
& Adequação \\
& Expressão hostil \\
Alucinações/ & Percepções falsas \\
Delírios/llusões & auditivas/visuais \\
Comportamento/Humor & Instável \\
& Não usual/bizarro/desinibido \\
& Motivação/energia \\
& Perambulação \\
& Depressão/Ansiedade relacionada \\
& Agitação/agressão/hostilidade \\
& Adequação/cooperação \\
& Insônia (tipo?) \\
Sono/Apetite & Atividade noturna \\
& Sonolência/hiper - hipossonia \\
& Apetite/alteração de peso \\
\hline
\end{tabular}

ESTUDO COOPERATIVO DA DOENÇA DE ALZHEIMER - IMPRESSÃO CLÍNICA GLOBAL DE ALTERAÇÃO (ADCS-CGIC) Página 8 de 9 ATIVIDADES DO COTIDIANO

\begin{tabular}{lll}
\hline DOMíNIO & INSTRUMENTOS DE SONDAGEM & OBSERVAÇÕES \\
\hline Capacidade funcional & Mobilidade \\
básica e complexa & Higiene/cuidados & \\
(instrumental) & Vestir-se \\
Praxias & Alimentar-se sozinho \\
& Fazer compras \\
& Tarefas domésticas/hobbies \\
& Finanças \\
Função social & Participação em: \\
& Interações sociais \\
& Atividades comunitárias \\
& Independência \\
& Fica desamparado \\
\hline
\end{tabular}

Notas e comentários:

Nome do examinador:

Iniciais do examinador:

Hora em que o teste foi aplicado: .

ESTUDO COOPERATIVO DA DOENÇA DE ALZHEIMER - IMPRESSÃO CLíNICA GLOBAL DE ALTERAÇÃO (ADCS-CGIC) Página 9 de 9 